\title{
SYPHILITIC EPIDIDYMITIS
}

\author{
By A. E. W. McLACHLAN, M.B., D.P.H.
}

THE observation, since I93I, of seven cases in which syphilitic epididymitis, ${ }^{*}$ without concomitant involvement of the body of the testis proper, occurred during the secondary or tertiary stages of a syphilitic infection, and the consequent examination of the literature, in which the rarity of this condition is emphasised, have led to the following report.

Three of the cases occurred during the secondary period, while the remaining four were recognised during the tertiary stages.

In addition to the cases reported here, in three further instances patients in the stage of late generalised syphilis presented lesions, the diagnosis of which was in all probability syphilitic epididymitis: in these cases the end result of treatment has not been observed.

\section{CASE RECORDS}

\section{Syphilitic Epididymitis Associated with Secondary Syphilis}

Case I.-Male, age 30. Exposure fourteen weeks previously: sore ten days later-healed spontaneously. Skin rash of four weeks' duration. No treatment.

On examination: healed sore with superimposed secondary moist papule on right side coronal sulcus. Moist papules on scrotum and inner aspects both thighs. Scattered pityriasiform rash on trunk, thighs, arms, chin, forehead. Moist papule inner aspect left cheek. Marked general adenopathy.

Epididymes : two nodules in head of left epididymis, three in head of right. Largest, size of small marble,

* The term epididymitis is used here to designate a morbid process affecting, clinically, the epididymis alone : involvement of other intrascrotal structures would be indicated by the use of the terms epididymo-vasitis, epididymoorchitis, etc., according to the structures involved. 
smallest, size of pea. Discrete, slightly tender on palpation. Remainder of epididymes clinically unaffected.

Testes and vasa showed no clinical alteration. No urinary symptoms. Urethral and prostatic smears and cultures showed no evidence of bacterial infection.

$S$. pallida demonstrated in puncture juice from nodule in right epididymis-puncture made through intact skin -and in serum from moist papule. Blood, Wassermann reaction ++ .

After two injections of "9I4," totalling $0.9 \mathrm{gm}$., and of bismuth $(0.8 \mathrm{gm}$.), the skin rash had markedly faded and the nodules in the epididymes were reduced to less than half the original size. After the third injection, patient was transferred, but continued treatment on a travelling card.

Reported five months later with arsenical jaundice. On examination, the nodules in both epididymes had completely disappeared. On questioning, patient stated that the skin rask had cleared up in " about a month," and that the epididymeal lesions had cleared in about the same time.

Case 2.-Male, aged 27. Denied any possibility of infection. Attended for examination because his child had died, aged six weeks, from syphilitic white pneumonia.

On examination: areolated, indurated sore in frenal area, with associated well-marked bilateral inguinal adenopathy. Early roseolar rash affecting trunk, arms and thighs. General adenopathy not marked.

Epididymes : nodule, size of marble in upper pole of right epididymis, one, size of pea, in upper pole of left epididymis. Discrete, not tender on palpation.

S. pallida ++ in serum from penile sore, and present in puncture juice from nodule in right epididymis. Blood, Wassermann reaction ++ .

No urethral discharge, but complained of some dysuria and marked diurnal and nocturnal frequency of micturition. Urine clear. Prostate slightly enlarged and acutely tender.

Urethral and prostatic smears showed only an occasional pus cell, and cultures a scanty growth of staphylococcus albus.

After three injections of " 9I4 " and liposoluble bismuth $(\mathrm{I} \cdot 65 \mathrm{gm}$. and $0.6 \mathrm{gm}$. respectively) the sore had healed, 
the skin rash faded and the epididymeal lesions had completely cleared up. The urinary symptoms also cleared up in this period without local treatment.

Case 3.-Male, aged 32. Exposure three to four months previously. Six to eight weeks later noticed " ulcers" on the penis: these disappeared with ointment. Recurrence of penile sores three weeks later, with associated skin rash.

On examination: moist papules on ventral aspect of body of the penis ; skin rash of impetigenous type, affecting chiefly nose, chin, submental area. Slight inguinal and general adenitis.

Left epididymis shows a diffuse even thickening, tense and elastic, involving whole epididymis-suggestion of nodulation in upper pole. Slightly tender. Discovered on routine examination-patient stated that he had only a vague feeling of local discomfort.

Testis and cord unaffected.

No urethral discharge. Urine clear, prostate normal. Urethral and prostatic smears showed an occasional leucocyte. Gr., F.T. negative.

$S$. pallida + in penile papules : blood, Wassermann and Kahn reactions ++ . Patient refused puncture of the epididymis.

After three injections of "9I4" (I.8 gm.) and liposoluble bismuth $(0.6 \mathrm{gm}$.) in thirteen days the body and tail of the epididymis had returned to normal : the head still showed some thickening and nodulation. One week after the fourth injections (total "9I4" $2.55 \mathrm{gm}$. and l.s.b. $0 \cdot 8 \mathrm{gm}$.) epididymis completely normal to palpation.

No clinical alteration of the testis throughout the period of observation.

\section{Syphilitic Epididymitis Associated with Tertiary Syphilis}

Case 4.-Male, aged 28. Sero-positive secondary syphilis three years previously. Attended for treatment for four months ("9I4," 4.35 gm., + Hg. grs. x), then ceased treatment.

For three weeks had noticed gradual swelling of the " testicles," increasing diurnal and nocturnal frequency of micturition, and a dragging and feeling of weight in the groins.

$$
136
$$




\section{SYPHILITIC EPIDIDYMITIS}

Frequency, two- to three-hourly, day and night. No dysuria.

On examination a bilateral, hard, craggy, nodular epididymitis was found, affecting the whole tissue. Slightly tender on palpation. Sharply demarcated from the testes and from the vasa.

Prostate slightly enlarged, tense, and markedly tender.

No other evidence of systemic infection.

Blood, Wassermann reaction ++ . S. pallida not demonstrated in puncture juice from epididymis. Urethral and prostatic smears showed no pus: culture showed a scanty growth of staphylococci and diphtheroids.

Under routine specific treatment the epididymeal lesions were reduced to half the original size by the fourteenth day, and after eight weeks treatment had completely resolved (" $9 \mathrm{I} 4$, ," $3 \mathrm{gm}$., bismuth, $2 \cdot 4 \mathrm{gm}$.).

In this period, without any local treatment, the urinary symptoms were completely relieved and the prostate showed no palpable abnormality.

The testes and the vasa showed no clinical alteration before, during, or after treatment.

Case 5.-Male, age 25. No history of infection. Reported because of difficulty in commencing act of micturition and partial incontinence.

During the immediately preceding three months patient had noticed a slowly increasing swelling of the right "testicle." Slightly tender on pressure, otherwise symptomless.

On examination: a hard, elastic, discrete nodule, the size of a large marble, was found in the upper pole of the right epididymis. Slightly tender on palpation. Testis, vas and prostate all showed no abnormality.

Urine showed a little pus, many calcium oxalate crystals, while culturally a poor growth of staphylococcus albus was obtained.

Blood, Wassermann ++ . Cerebrospinal fluid, W.R. ++ , cells, 95 per c.mm., globulin markedly increased, goldsol II2332IOOO.

In view of the neurosyphilis, treatment was commenced with tryparasamide and bismuth.

After six weeks' treatment (tryparasamide, I2 gm., and liposoluble bismuth, I.2 gm.) there was complete resolution of the epididymeal lesion. Relief of the urinary 
symptoms was almost complete at the end of three months treatment.

Case 6.-Male, age 43. Unaware of any infection. For four months had noticed "lumps on back and thigh." For " about a month " had noticed slight swelling and dragging pain in the right testicle.

On examination subcutaneous gummata were found over the eighth rib in the posterior axillary line and on the outer aspect of the left thigh four inches below the great trochanter, the latter with early skin involvement. The right epididymis showed a diffuse thickening, with two nodules the size of large peas in the upper pole. Testis clinically normal. Left scrotal contents normal. No urethral discharge, no clinical or bacteriological evidence of tuberculous or other bacterial infection of the urinary tract.

Treatment was commenced with neoarsphenamine and liposoluble bismuth. After eight weeks (total, 2.8 gm. "9I4" and 1.s.b. $0.8 \mathrm{gm}$.) the gummata and the epididymeal lesions had completely resolved. The testis remained clinically unaltered throughout treatment.

Case 7.- Male, age 35. Unaware of any infection. For five weeks had noticed a "lump in the testicle," gradually increasing in size. No dysuria, no frequency of urination, no other symptoms referable to the genitourinary tract.

On examination a hard nodule the size of a small walnut was found in the upper pole of the left epididymis. Non-tender, separated from body of the testis, of cartilagenous hardness, with suggestion of lobulation, extending somewhat into the body of the epididymis and overlapping, if not actually involving, the adjacent portion of the vas deferens. No signs of urinary infection. Prostate smooth and of normal consistence.

Blood, Wassermann and Kahn reactions ++ .

After four injections of " $9 \mathrm{I} 4$ " and bismuth (I.95 gm. and $0.8 \mathrm{gm}$. respectively) the nodule had decreased to one-half to two-thirds of the original size. After the first course (4.35 gm. "9I4" and 2 gm. bismuth in ten weeks) the nodule had decreased to the size of a large pea. Rested for four weeks, to take Potass. Iod., grs. xv t.d.s. for the first fortnight. Reported at end of fourth week with jaundice. Nodule now the size of a small pea, with slight angularity towards vas. 


\section{SYPHILITIC EPIDIDYMITIS}

Further treatment over several months has produced no further diminution in the size of this nodule.

\section{HistORICAL}

Astruc (I736) recorded that in certain cases of syphilis, as opposed to gonorrhœa, lesions had been noted in the epididymis. Benjamin Bell (I793) reported the extension to the epididymis of luetic orchitis in cases where the systemic disease had been neglected, or where, the ætiology being unrecognised, the patient had not been treated with mercury. Ricord (I838) considered that the epididymis could be the seat of syphilitic deposits, but in I 845 suggested gonorrhœa as the localising agent in a case where a syphilitic orchitis commenced with lesions in the epididymis. Astley Cooper (I84I) recognised the involvement of the epididymis in syphilitic lesions of the testicle. He records one case in which syphilitic orchitis commenced with a lesion in the epididymis.

Hélot (I846), Gosselin (I848), Maisonneuve and Montanier, Follin, Nélaton (I852), Curling (I857), and others all considered that the epididymis might be involved by extension from a syphilitic orchitis. Virchow (I859) stated that the epididymis was infrequently involved in secondary syphilis, but escaped implication in the tertiary stage.

Dron (1863) was the first to emphasise that the epididymis could be involved by a syphilitic process without concomitant involvement of the body of the testis proper. In his report Dron publishes I6 cases : 2 of these are, however, cases of epididymo-orchitis. The remaining I4 showed lesions of the epididymis unaccompanied by clinical involvement of the testis. Six cases of the series gave a history of antecedent gonorrhœea : of these, four had shown no epididymitis. The gonorrhœa had in all cases been cured for several months or years before the onset of the syphilitic epididymitis. The diagnosis was made by exclusion of gonococcal, tuberculous or other bacterial infections, and confirmed by the therapeutic test.

Later observations by Lancereaux (I866), Fournier (1875), Balme (I876), Pinner (I884), Arthur Cooper (I885), Jacobson (I893), Comby (I899), v. Zeissl (I902), Micheel (I906), Hutchinson (I9I3), Wright (I9I6), Lisser and 


\section{BRITISH JOURNAL OF VENEREAL DISEASES}

Hinman (I9I8), Michelson (I9I9), Rolnick (I924), le Fur (I93I) and others have confirmed the accuracy of Dron's statements.

\section{InCidence AND Period of Involvement of THE EPIDIDYMIS}

Dron noted involvement of the epididymis in the course of systemic syphilis in I6 patients out of a total of 200 . Balme found $I_{3}$ instances in a total of 2,300 syphilitics. The 7 cases reported here were recognised from among a total of I,200 male patients suffering from syphilis.

According to Dron, the epididymis is involved from the first to the fifth month following the appearance of the secondary rash: the longest interval in the series was four years after infection. Gosselin considered that the epididymis was never involved in less than three and a half months from the date of infection. v. Zeissl reported the occurrence of a syphilitic epididymitis, accompanied by a papular syphilide, in the second month after infection. In Fournier's series, 8 patients showed this complication four to five months after development of the chancre, 6 between the fifth and fourteenth month, 8 between the second and eighth years, and I fifteen years after primary infection.

Of Michelson's 7 cases, one was a child of two years, suffering from congenital syphilis ; in two instances the epididymitis occurred one year after infection; while in the others the intervals were four, five, seven and seven years respectively. In the case reported by Lisser and Hinman the epididymis was affected after an interval of nine years; while one of Wright's patients developed a luetic epididymitis seventeen years after the primary infection.

In only three of the cases reported here is the time of incidence ascertainable: Case I, fourteen weeks after infection; Case 2, three to four months after infection; and Case 4, approximately three years after a partially treated seropositive secondary syphilis.

Rolnick suggests that the frequent localisation of the lesion in the head of the epididymis is due to the direct blood supply of the globus major from a branch of the internal spermatic artery given off just before the main stem entered the testis - an explanation put forward by 
Jacobson in 1893 to account for the vascular spread of a tuberculous infection to the head of the epididymis.

Belfield, however, offers another explanation, that the testes have an excretory function and that the epididymes and vasa are the excretory tubules of the testis; thus spirochætes excreted by the testis may pass through the testis without injuring this structure and lodge in the epididymis, causing pathological sequelæ.

\section{Symptoms, Signs and Clinical Course}

In the secondary stage of syphilis, lesions of the epididymis are transient and closely follow the course of the skin rash, disappearing spontaneously even in the absence of treatment. Almost invariably symptomless, these lesions may be accidentally discovered by the patient or revealed on clinical examination. Fournier and Balme have, however, reported instances in which local pain and tenderness, at the onset of a syphilitic epididymitis, were distinctive features, while Dron records that in such cases nocturnal exacerbations of pain may be expected.

The epididymis, rarely, may be involved before the appearance of the skin rash. McDonagh (I920) records such a case in which " . . . a testicle was removed for a supposed tubercular epididymitis, the generalised papular rash not making its appearance till the patient was convalescing from the operation."

In tertiary syphilis the onset of an epididymitis is insidious and symptomless. The swelling may be accidentally noticed by the patient, or, if the condition progresses in the absence of treatment, may only be noticed when a feeling of weight in the testicle, dragging along the line of the spermatic cord, or the development of a hydrocele direct attention to the underlying lesion.

In the majority of cases the site of the lesion is in the globus major, less frequently in the body, in the globus minor, or involving the entire epididymis. In the lastmentioned type, the globus major invariably shows the most marked changes.

The lesions seldom attain any great size, in general varying from the size of a small pea to that of a large olive, while in consistence they vary from a tense elasticity to a cartilagenous hardness. Partial fusion of the 
individual lesions may give the impression of lobulation. McDonagh likens palpation of the affected pole of the epididymis to feeling a bunch of grapes through a soft bag. This accurately describes the feel of the nodules observed in cases of " secondary syphilitic " epididymitis, but does not adequately represent the cartilagenous density and irregular nodularities of " tertiary syphilitic" epididymitis.

The affected areas are sharply defined and differentiated from the unaffected part of the epididymis and from the testis proper. Where the entire epididymis is involved there is, in the early stages, a smooth, tense, elastic thickening of approximately the size of a pencil, later going on to nodule formation : the sharp line of demarcation between the epididymis and the body of the testis gives the illusion of a helmet-shaped epididymeal mass in apposition to, but not overlapping, the body of the testis.

One epididymis only may be affected, the second may subsequently be implicated, or both may be involved simultaneously.

Extension of the inflammatory process from the epididymis to the testis has been recorded (Astley Cooper), to the spermatic cord (v. Zeissl, Wright, Michelson, et al.) ; Fuller (I900) has emphasised the marked tendency to extension into the parietal layer of the tunica vaginalis, " the result often being that the structure becomes thickened and rigid, the extent of the inflammatory process being marked by a crusty ridge, which I have likened in feel to that of an oyster shell. Associated with this secondary involvement of the tunica vaginalis is hydrocele." . . . "So prominent in fact are the features of hydrocele, and thickening of the tunica vaginalis in these cases that d'Oelsnitz (I886) and Ozenne (I888) have overlooked the starting point of the inflammation and have classed these cases as ones of syphilitic pachyvaginalitis."

The association of hydrocele with syphilitic epididymitis has been remarked by many observers.

Sinus formation may occur, as in the cases reported by Michelson, Berthole (I868) and others.

In Berthole's case the sinuses were very persistent and simulated tubercle: cure, however, followed antisyphilitic medication.

In congenital syphilis the epididymis may show localised syphilitic deposits with a clinically unaffected 
testis, as in the cases reported by Greenfield (I887), Wilks (I882), Comby (I889), Michelson and others.

Resolution may commence at any stage of the process, even in the absence of treatment, with complete return to normality, or, more commonly, some slight fibrous residual thickening of the epididymis. Pinner (I884) states that in some cases the epididymis becomes atrophic, with an occluded lumen.

Few pathological observations have been recorded with respect to syphilitic epididymitis. That the changes are interstitial is supported by the observation of Dron that the ejaculate of a patient suffering from bilateral syphilitic epididymitis showed spermatozoa, while one of Wright's patients successfully impregnated his wife after the removal of one testis when a hydrocele associated with a syphilitic epididymitis had developed on the other side. Gross (I88I), on the other hand, recorded a case of syphilitic epididymitis in which spermatozoa were absent from the semen.

\section{Diagnosis}

In establishing a diagnosis of syphilitic epididymitis several criteria should be fulfilled :-

(I) Exclusion of gonorrhœal, tuberculous, or other bacterial ætiology.

(2) Exclusion of cysts, or of malignant disease of the epididymis.

(3) Presence of evidence-serological or clinical-of associated syphilis. Demonstration of $S$. pallida in epididymeal lesion.

(4) Complete resolution of the lesion under anti-specific treatment.

(5) No clinical alteration of the testes during the period of observation.

The generally insidious onset of a syphilitic epididymitis - with the production of an indolent, elastic lesion of small volume, smooth, but not infrequently irregular, affecting typically the globus major, less typically affecting the body, the tail, or the entire epididymis - in association with other signs of syphilis, differs greatly from the acute generally febrile onset of a gonococcal or bacterial epididymitis. The routine investigation of the various secretions and exudates should complete the differentiation. 
Demonstration of $S$. pallida in puncture fluid from the epididymis proved easy in two cases associated with secondary syphilis : this, however, may prove difficult in tertiary lesions, in which the organisms are infrequent.

The acute form of tuberculous epididymitis should present few difficulties: the subacute type, however, characterised by slow and insidious onset, may in the early stages be clinically indistinguishable from the syphilitic. Hutchinson quotes the case of a patient who seven and a half years after a primary infection "... came with a lump as big as a small hazel nut in one epididymis. It felt as much like tubercle as gumma, but it vanished under specific treatment, so that there could be no real doubt as to its real nature," while excisions of syphilitic epididymes, erroneously assumed to be tuberculous, have been reported by Arthur Cooper, Wright, McDonagh and others. The history, physical signs, constitutional evidences of disease, and the results of pathological investigations must all be considered. If there still remains doubt the therapeutic test should be applied.

Cysts generally affect the upper pole of the epididymis, and are softer and less elastic. Fluctuation may be detected, and translucency. Rare before the age of 40 . In cases of doubt aspiration may be resorted to.

Benign growths of the epididymis are practically unknown. Bailey (I936), referring to malignant disease of the epididymis, states, "in practically every case which has been reported the original diagnosis was one of chronic epididymitis. . . ." The course is often slow at the outset, but rapid metastases may arise after injury. Exclusion of other causes of an epididymitis should suggest the consideration of possible malignancy. The occurrence of malignant disease in association with syphilis may have to be considered : apparent improvement of a malignant lesion has been frequently observed under anti-specific treatment, and unless the patient is carefully observed operation or other treatment may be too long deferred. In a case seen some three years ago, the patient reported with a left epididymo-orchitis. Wassermann and Kahn reactions proved strongly positive. Treated with "9I4" and bismuth. Testicle improved markedly, but had not returned to normal after the first course. Patient developed an arsenical dermatitis, after which treatment was continued with bismuth 
alone. Attendance somewhat irregular. Testicle remained small, but never regained normal sensation. Thirteen months from date of first reporting, complained of diarrhœa which had persisted for two months. On examination a mass was found in the left hypochondrium, while the testicle had recently enlarged markedly in size. Refused admission. Died one month later after operation for " intestinal obstruction."

In cases, therefore, where there is real doubt as to possible malignancy surgical exploration should be carried out.

A true syphilitic epididymitis should resolve speedily under the influence of modern treatment. In the majority of cases, resolution is complete and the epididymis restored, clinically, to normal. In some few cases, however, it is probable that a small residual fibrous nodule may be left to mark the site of a previous gummous epididymitis, as in Case 7.

\section{Prognosis AND Treatment}

No prognostic gravity attaches to a syphilitic epididymitis : it is simply a local manifestation of a generalised infection.

Local treatment is symptomatic-support, tapping of hydrocele, etc., as indicated. The response to routine antispecific treatment is rapid and satisfactory.

\section{SUMMARY AND CONCLUSIONS}

Seven cases of syphilitic epididymitis without concomitant involvement of the body of the testis proper are reported : of these, three occurred in the secondary stage and four in the tertiary stage.

$S$. pallida was demonstrated in the puncture juice from the epididymeal lesions of two of the three cases associated with secondary syphilis. In the third case puncture was not carried out. In the only case of tertiary syphilitic epididymitis punctured $S$. pallida was not found.

A review of the literature emphasises the rarity of the condition : many of the observers, however, express the view that syphilitic epididymitis is less uncommon than the number of published cases suggests.

The rarity of recognition of syphilitic epididymitis in 
association with secondary syphilis is probably accounted for by the symptomlessness of the condition, the earlier diagnosis of primary and secondary syphilis and the greater efficacy of modern arsenical treatment.

More instances of tertiary syphilitic epididymitis would in all probability come to light if syphilis were considered as one of the possible ætiological factors of epididymitis, and in cases of doubt the result of a therapeutic test observed.

I have to express my indebtedness to Mr. A. E. Roche for reference of clinical material, to Mr. F. C. Endean for clinical facilities afforded, and to Dr. David Thomson for access to much of the literature.

\section{BIBLIOGRAPHY}

Astruc, J. (1763): Traité des Maladies Vénériennes, 1, 296.

Bailey, Hamilton, and Matheson, N. M. (I936): "Recent Advances in Genito-Urinary Surgery," 82.

BaLMe (1876): Thèse de Paris.

BeLfield (I9I2): J. Am. Med. Assn., October Igth.

BELL, Benjamin (I793): "A Treatise on Gonorrhœa Virulenta and Lues Venerea."

BERTHOLE (1868) : Union méd., 1, 57.

Comby (1889): Ann. de Derm. et de Syph.

COOPER, AlFred (1895): "Syphilis," 2nd ed., London, 258.

COOPER, ARTHUR (1885): Brit. Med. J., $i$, ro94.

CoOPER, Astley (I84I) : "Diseases of the Testicle," London, and ed., 167.

CuRLING, T. B. (I857) : "A Practical Treatise on Diseases of the Testicle."

Dron, A. (1863) : Arch. gén. de Med., 2, 513, 724, November and December.

Fournier, A. (1875): Ann. de Derm. et de Syph., 6, 224.

FULlER, E. (I9O0) : "Diseases of the Genito-Urinary System."

LE FUR, R. (I93I) : Bull. d'urol., Paris, 5, I76.

Gosselin, L. (1848) : Arch. gén. de Méd., 16, r63, Fev.

GREENFIELD, W. G. (I877), "Pathological Transactions," 225.

Gross, S. W. (188I) : "On Impotence," 93.

HÉLoT (1846): J. de Chir., 4, ro6.

Hutchinson, Jonathan (I896): "Syphilis," 269.

JACOBSON, W. H. A. (I893): "Diseases of the Male Organs of Generation," 3II, 33I, 348 .

LANCEREAUX, E. (I868) : "Treatise on Sỷphilis," New Sydenham

Soc. translation, 1, 192, 272; 2 , I49.

Lisser, H., and HINMAN, F. (1918): Am. J. Syph., $2,465$.

McDonAGH, J. E. R. (I920) : "Venereal Diseases," 85.

MiCHEEL (Ig06): " Dissertation," Rostock.

Michelson, H. E. (I9I9) : J. Am. Med,Assn., 73, No. I9, I43I, November 8th.

$$
146
$$


Nélaton (I852) : Gaz. des Hôp., Paris, No. 42, I65, 8 Avr. D'Oelsnitz, A. (I886) : Thèse de Paris.

Ozenne (I888) : Gaz. Hebdom. de Méd., 25, 196.

Pinner, O. (I884): Berl. klin. Wchnschr., 41, 655, October I3th. Réclus, P. (1883): Gaz. Hebd. de Méd., 20, 2, I7, January I2th. RICORD (I838): Traité pratique des Maladies Vénériennes. RICORD (1845): Gaz. des Hôp., Paris.

Rolnick, H. C. (I924): J. Urol., 12, I47, August.

VIRCHOw, R. (I859): Syphilis Constit., trad. franç.

WILKS, S. (I882) : Brit. Med. J., $i, 425$.

WRIGHT, F. R. (I9I6): Urol. and Cutan. Review, 20, 12, 66r.

VON ZEISSL, H. (I9O2) : Lehrbuch der venerisch. Krankheiten, 4I7. 